\title{
Design of control software for a high-speed coherent Doppler lidar system for $\mathrm{CO}_{2}$ measurement
}

\author{
Randal L. VanValkenburg ${ }^{1}$, Jeffrey Y. Beyon², Grady J. Koch ${ }^{3}$, \\ Jirong $\mathrm{Yu}^{4}$, Upendra N. Singh ${ }^{5}$, and Michael J. Kavaya ${ }^{6}$ \\ ${ }^{1}$ NASA Langley Research Center, MS 472, Hampton, VA 23681, USA, \\ Randal.L.VanValkenburg@nasa.gov \\ ${ }^{2}$ NASA Langley Research Center, MS 488, Hampton, VA 23681, USA, Jeffrey.Y.Beyon@nasa.gov \\ ${ }^{3}$ NASA Langley Research Center, MS 468, Hampton, VA 23681, USA, Grady.J.Koch@nasa.gov \\ ${ }^{4}$ NASA Langley Research Center, MS 468, Hampton, VA 23681, USA, J.Yu@nasa.gov \\ ${ }^{5}$ NASA Langley Research Center, MS 468, Hampton, VA 23681, USA, \\ Upendra.N.Singh@nasa.gov \\ ${ }^{6}$ NASA Langley Research Center, MS 468, Hampton, VA 23681, USA, \\ Michael.J.Kavaya@nasa.gov
}

\begin{abstract}
The design of the software for a 2-micron coherent high-speed Doppler lidar system for $\mathrm{CO}_{2}$ measurement at NASA Langley Research Center is discussed in this paper. The specific strategy and design topology to meet the requirements of the system are reviewed. In order to attain the high-speed digitization of the different types of signals to be sampled on multiple channels, a carefully planned design of the control software is imperative. Samples of digitized data from each channel and their roles in data analysis post processing are also presented. Several challenges of extremely-fast, high volume data acquisition are discussed. The software must check the validity of each lidar return as well as other monitoring channel data in real-time. For such high-speed data acquisition systems, the software is a key component that enables the entire scope of $\mathrm{CO}_{2}$ measurement studies using commercially available system components.
\end{abstract}

Keywords: Coherent Lidar, Data Acquisition, Differential Absorption Lidar, Software, $\mathrm{CO}_{2}$ Measurement

\section{INTRODUCTION}

Over the past decade or so, extensive research and development has been conducted by the NASA Langley Research Center to develop and mature high energy, pulsed, 2-micron laser technology. These lasers have been utilized in both Doppler Lidar ${ }^{2-6,8,10-13}$ and Differential Absorption Lidar (DIAL) ${ }^{7,9}$ configurations, using both direct and coherent (heterodyned) detection techniques.

Our current $\mathrm{CO}_{2}$ Measurement Demonstration makes use of a high-speed coherent Doppler lidar ${ }^{1}$ used previously for wind measurements. Three significant improvements have been made in the current transceiver compared to the system used in the ground demonstration in 2007. First, the system is now a $10 \mathrm{~Hz}$, on-and-off pulse pair format transmitter, allowing improved measurement precision by sensing nearly the same atmospheric volume for both the on-line and offline pulses, and effectively increasing the pulse repetition rate by four-fold. This will improve the measurement precision to better than $1.5 \%$ using $1000-\mathrm{m}$ range bins, and reduce the required integration time to 2.112 minutes. Second, the selection of the $\mathrm{R} 32 \mathrm{CO}_{2}$ absorption line over R22 further improves the measurement precision, due to its negligible water vapor line interference. The parameters of the R32 line are given with accuracy of $0.4 \%$ for the line strength, $0.15 \%$ for the air broadening coefficient, and $0.45 \%$ for the temperature parameter. These accuracies, together with the assumed accuracy of $2.6 \%$ for the pressure shift, are needed to attain the required $\mathrm{CO}_{2}$ measurement precisions. Third, the laser power is eight times greater than that of the previous ground demonstration system. It will cover a larger measurement distance with increased signal carrier to noise ratio (CNR). 
A major advantage of our current lidar is its ability to serve as a dual-use instrument - measuring atmospheric $\mathrm{CO}_{2}$ concentrations as a coherent DIAL instrument, and 3D winds as a coherent Doppler lidar instrument. The capability to make such complementary measurements, coincident in space and time and with improved precisions and accuracies, is key to enabling future strategic atmospheric measurements, such as those called for in the NRC Decadal Surveys.

This paper will discuss various aspects related to the development of the Data Acquisition (DAQ) Software (SW) for the $\mathrm{CO}_{2}$ Demonstration, and its operation and functionality; including hardware (HW) configuration, scanner operation, data acquisition, and minimal data processing and storage of the acquired signals. It should be stressed that this research effort - to show the feasibility of using an existing coherent Doppler lidar system to measure atmospheric $\mathrm{CO}_{2}$ - was a technology demonstration, requiring quick modification and turnaround of the control software. Software development for operational science instruments, and especially flight instruments, is governed by NASA Procedural Requirements that ensure adherence to documented development processes.

This paper is organized as follows: the software development environment and the acquisition hardware are described briefly in Section 2. Section 3 provides an overview of the DAQ software and its user interface architecture, with its Validation Lidar (VALIDAR) heritage. Section 4 describes the acquired data and ways to assess the quality of each lidar return pulse. The last section provides conclusions as well as suggestions for future research and development of software for these dual use coherent lidar instruments.

\section{THE ACQUISITION HARDWARE AND ENVIRONMENT}

The data acquisition electronics were housed in an Agilent Technologies Acqiris CC105 Compact PCI (cPCI) Crate which provides five horizontal 6U slots. In the lowest "system" slot was a Single Board Computer (SBC) hosting a Microsoft Windows environment, allowing SW development and maintenance using the system itself.

We used two identical Agilent Acqiris DC-252 10-bit Series cPCI/PXI Digitizers cards. Each DC-252 was equipped with a mezzanine front-end card that provided standard (50 Ohms) impedance coupling through three BNC connectors: one for each of its two input channels, and another for an external trigger signal. Internal triggering is also supported, using the signal level on one of the input channels. Both of our DC-252 cards were equipped with the maximum optional memory, accommodating up to 256M samples per channel. While it may be tempting to install electronics cards in alternate slots for thermal reasons, the DC-252 cards were placed in the two adjacent slots just above the system slot to minimize potential communications delays.

The DC-252 digitizers support a large number of configurations for different data acquisition requirements. For example, both Channel 1 and Channel 2 have their own A/D converter (ADC) and acquisition memory, but the ADCs can be "interleaved" and the acquisition memory combined to support a single channel with more capability. In addition, the acquisition memory is dual-ported and can be configured as a circular buffer to allow for Simultaneous Acquisition and Readout (SAR) operation. These advanced features were not required for the current demonstration project, but may be useful for future enhancements.

In our configuration, the two DC-252 Digitizers were connected together using an external AS bus 2 connector. The Acqiris System Software configures the two identical dual-channel digitizers into a single "Multi-Instrument" with four input channels, and which share a common internal clock and external trigger. We used one input channel (Channel 1) of each DC-252 card. Note that for the 4-channel Multi-Instrument, the system software renumbers the channels, and they are henceforth referred to as Channel 1 and Channel 3.

The Acqiris System Software Application Programming Interface (API) is described in a variety of documents such as a Programmer's References Manual ${ }^{14}$ and a User Manual ${ }^{15}$ for the 10 -bit digitizer family. Some API procedures are specific to the 10-bit series while others are applicable to different models. In some cases different models use the same procedures but interpret the parameters passed to them differently. It is essential that the software developer read the documentation thoroughly. 
$\mathrm{CO}_{2}$ measurements require very large amounts amount of data to be acquired in a short time. It was thus necessary to test the HW to ensure that it could accommodate these sample frequencies and data volumes, without dropping a pulse. The DC-252 digitizers store each triggered pulse in its own "segment" of acquisition memory, along with a Segment

Descriptor - a data structure containing timing and auxiliary information. Each Segment Descriptor includes two 32-bit unsigned integer variables, timeStampHi and timeStampLo, that record the pulse's start time in picoseconds. As a stress test, we acquired 75,000 samples at $500 \mathrm{MHz}$ for each of 200 pulses fired at $5 \mathrm{kHz}$, corresponding to $200 \mu \mathrm{s}$ or 200,000,000 ps between pulses. If no pulses were missed, the timestamps between the first and last pulse should differ by about $199 * 200 \mu \mathrm{s}=39,800 \mu \mathrm{s}$. The segment descriptors for the first and last pulses showed the following:

\begin{tabular}{lrrr}
\multicolumn{2}{c}{ segDesc[0] } & \multicolumn{2}{c}{ segDesc[199] } \\
timeStampHi & 5972 & timeStampHi & 5982 \\
timeStampLo & 3615426219 & timeStampLo & 465744444
\end{tabular}

The less significant timeStampLo "rolls over" at $2^{32} \mathrm{ps,} \mathrm{incrementing} \mathrm{timeStampHi} \mathrm{in} \mathrm{the} \mathrm{process.} \mathrm{Thus,} \mathrm{the} \mathrm{first} \mathrm{and} \mathrm{last}$ times stamps are equivalent to 25,653,160,117,931 ps and 25,692,960,109,116 ps, respectively, and differ by $39,799.991185 \mu \mathrm{s}$. This indicates that the digitizer captured all of the pulses, even at a $5 \mathrm{kHz}$ pulse rate and while collecting 75,000 samples per pulse.

\section{DESIGN AND DEVELOPMENT OF $\mathrm{CO}_{2}$ MEASUREMENT SOFTWARE}

We developed the Data Acquisition (DAQ) Software to support this Coherent Lidar $\mathrm{CO}_{2}$ Measurement Demonstration by modifying the existing VALIDAR application written in 2007 by the second author of this paper. The VALIDAR SW was developed using Visual C++ 6.0. The Graphical User Interface (GUI) was built within Microsoft Visual Studio, and made use of National Instruments Measurement Studio for graphical displays (Figure 1).
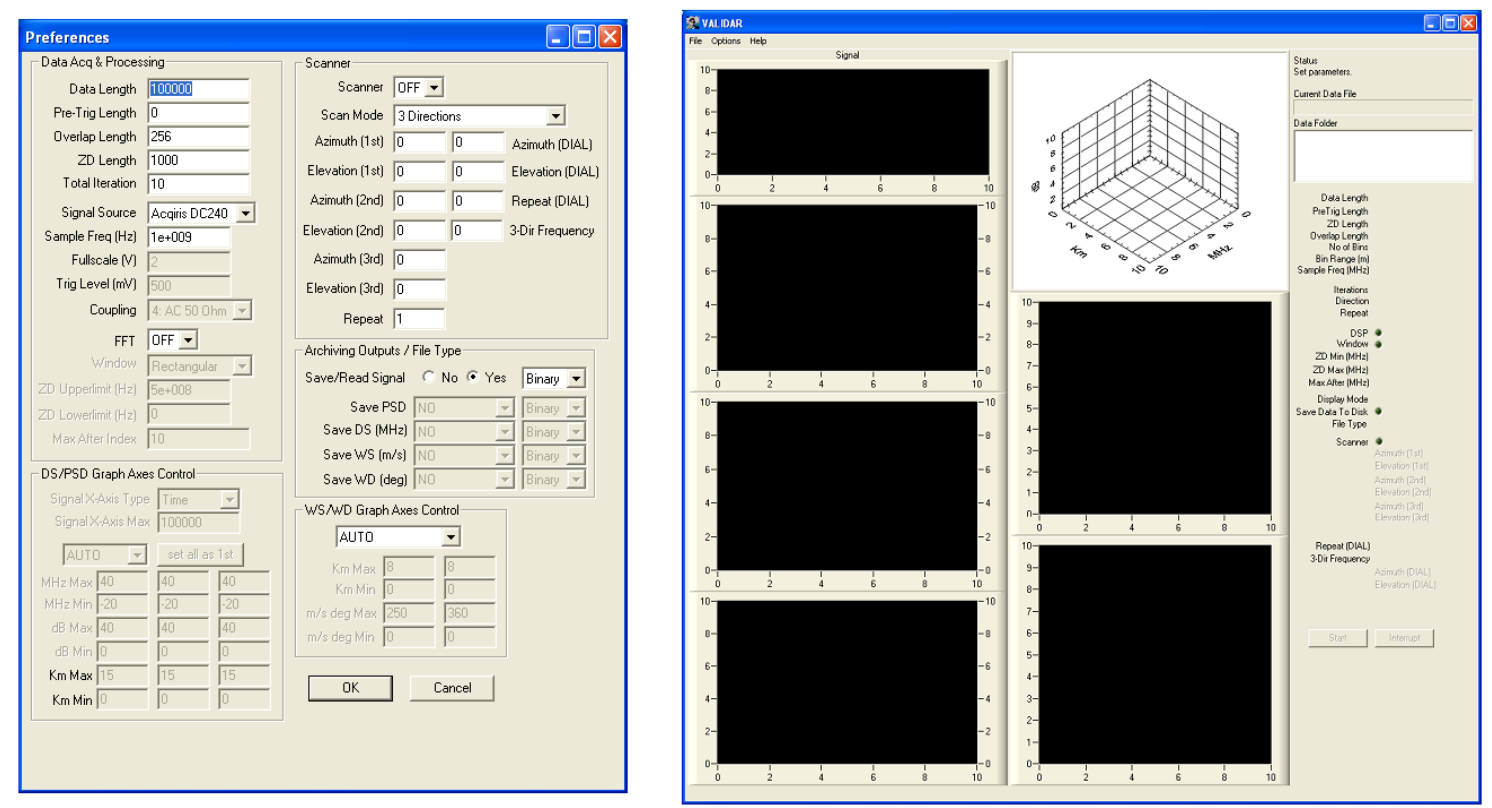

Fig. 1. The Original VALIDAR Preferences Dialog and Run-Time Display

We opted to use the same tools and versions used to develop the VALIDAR SW for several reasons. 1) This precluded some potential problems, such as compiler inconsistencies. 2) The $\mathrm{CO}_{2}$ and Doppler wind measurements - while different in nature - used essentially the same laser equipment, allowing reuse of the scanner control software without modification. 3) Our $\mathrm{CO}_{2}$ Demo used new digitizer $\mathrm{HW}$, but it was from the same vendor and the software interfaces were very similar. Finally, we hoped to facilitate future development of SW to acquire both types of measurements. 
There were also some challenges presented by these choices. The VALIDAR SW included DAQ, but also Data Analysis and Processing (DAP) functionality - which was relegated to post-processing for the $\mathrm{CO}_{2}$ Demo. Also, a significant fraction of VALIDAR code implemented its User Interface, and modifications to this code were not straightforward.

The architecture of the $\mathrm{CO}_{2}$ Demo SW was built around the two VALIDAR Windows Dialogs (Figure 1), implemented as Visual C++ classes. User settings for a data acquisition are read from an ASCII preferences file, displayed in the Preferences Dialog (left) where they can be modified, then re-saved to the ASCII file. When the OK button on the Preferences Dialog is clicked, the Main Dialog (right) is enabled for data acquisition.

We initially planned to modify the VALIDAR SW and GUI, but retain all of its Doppler wind features. However, the current software developer was not familiar with the internals of the VALIDAR C++ code. To make the amount of code manageable for understanding and modification within the short deadline, it became necessary to temporarily strip out the unused Doppler code and interface elements.

\section{DATA ACQUISITION AND ANALYSIS}

For the $\mathrm{CO}_{2}$ Demonstration, we acquired data using the following parameters:

- Sampling Frequency $500 \mathrm{MHz}$

- Samples per Pulse 17,500
- Pulses per Set/File

- Pulse Frequency
600

$10 \mathrm{~Hz}$

The digitizer HW was configured to simultaneously acquire data on two channels. The data acquired for two consecutive pulses are displayed in Figure 2, with Channel 1 in the top graph and Channel 3 in the bottom graph. The first 1024 samples of each pulse are called the Monitor Region and the remaining samples the (Atmospheric) Return Region. (For Doppler wind measurements the Monitor Region is referred to as the Zero Doppler (ZD) Region.)
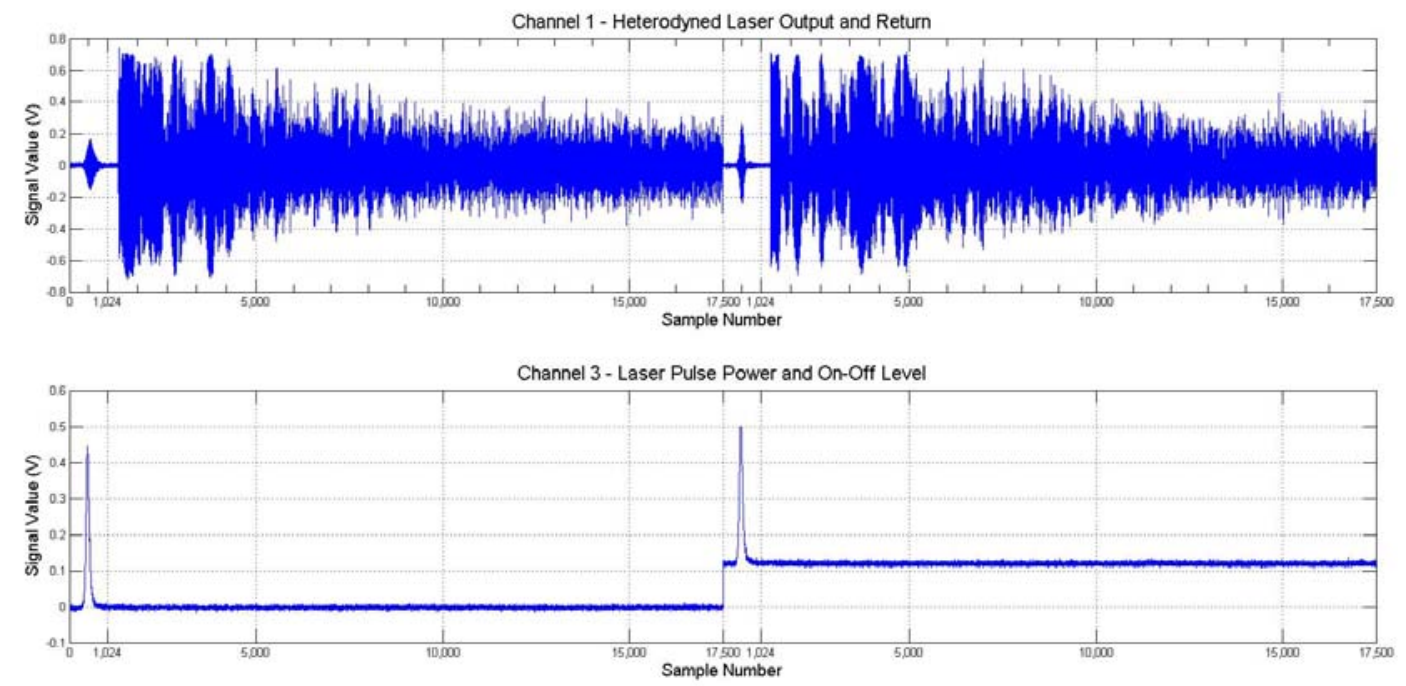

Fig. 2. Two consecutive pulses showing acquired data from both Channel 1 (heterodyned lidar pulse, outgoing and atmospheric return) and Channel 3 (pulse represents outgoing lidar pulse power, base signal represents input to switch multiplexing on-line and off-line frequencies). For this $\mathrm{CO}_{2}$ Demo the laser was pulsed at $10 \mathrm{~Hz}$, and 17,500 samples acquired at $500 \mathrm{MHz}$ for each. The first pulse is at the on-line frequency and the second at the off-line frequency.

The Monitor Regions of both channels are expanded in Figure 3. The first pulse was at the on-line frequency of the DIAL instrument and the second at the off-line frequency. (Note that on-line denotes a frequency selected within the R32 $\mathrm{CO}_{2}$ absorption line, while off-line denotes one selected nearby - but well outside the absorption line. The on-line frequency may coincide with the center frequency of the absorption line, but more often is slightly displaced from the 
center at a side-line frequency.) By taking an FFT (Figure 4) of the Channel 1 Monitor Region, and finding the peak frequency, one can determine the quality of each pulse. In our experiment, a pulse was deemed Good if the peak frequency was within $5 \mathrm{MHz}$ of the average frequency, which was about $109 \mathrm{MHz}$. In one session we collected 30 sets of 600 pulses each (18,000 pulses) while observing only 14 Bad pulses.
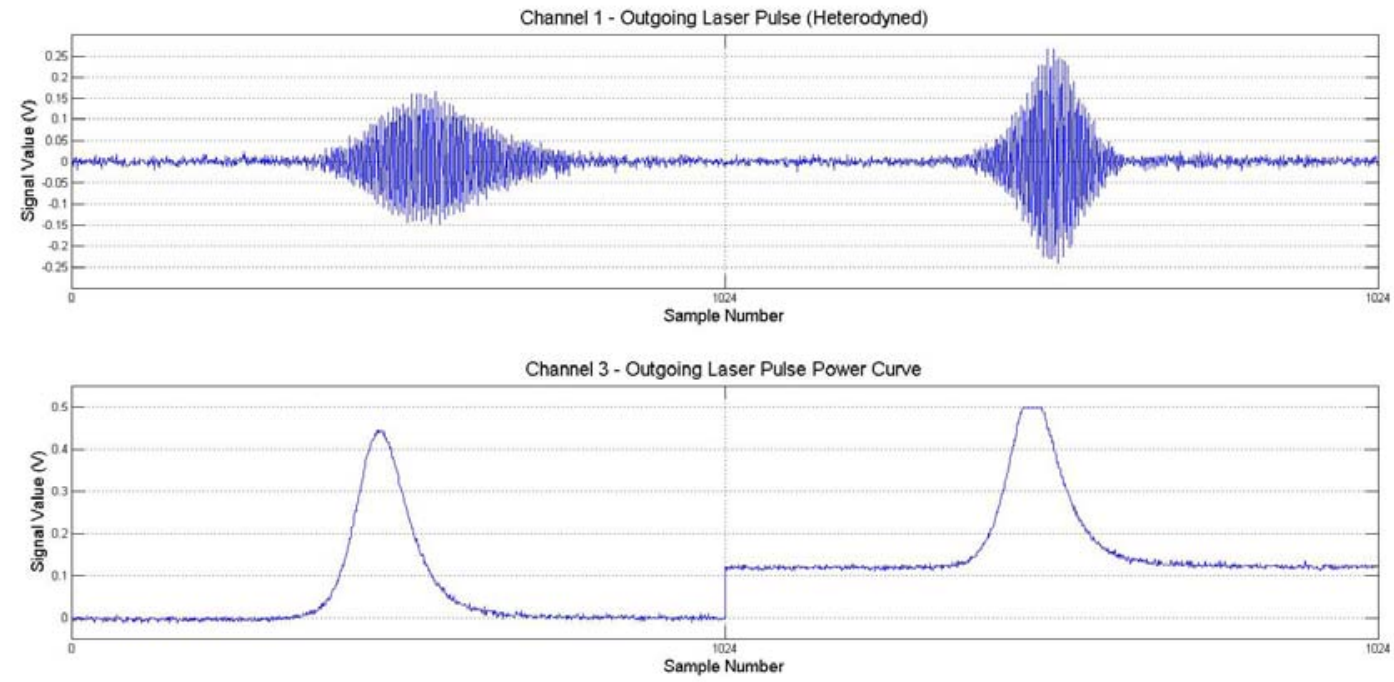

Fig. 3. Expanded view of the Monitor Region for the same two pulses as in Figure 2. The Channel 1 monitor data (top) provides frequency spectrum information for the corresponding lidar pulse as it is emitted. This is used to identify Good vs. Bad pulses, and also to quantify residual jitter between the frequencies of the seed laser signal and the actual lidar pulse. The base signal level in the Channel 3 monitor region (bottom) indicates whether the pulse frequency is online or off-line. (On-line is indicated by the lower level, near $0.0 \mathrm{~V}$.) The area under the peak can be integrated to determine total pulse power. Note the slight saturation of the second pulse where the Channel 3 data are truncated at about $0.5 \mathrm{~V}$. This is easily corrected using the Acqiris System Software to adjust the offset for that digitizer channel.

The VALIDAR program implements both DAQ and DAP Tasks. For the $\mathrm{CO}_{2}$ Demo, DAP was delegated to postprocessing using separate software. However, we prototyped some of the $\mathrm{CO}_{2}$ DAP SW in MATLAB, pending additional resources to develop more formal versions.

While the $\mathrm{CO}_{2}$ Demo was restricted to DAQ SW, some DAP SW functionality is useful to determine the quality and characteristics of each lidar return pulse during operations. This information can facilitate decisions related to where to take subsequent data and what acquisition settings to change. In addition, while pulses nominally alternate between online and off-line frequencies, occasionally 2 or 3 adjacent on-line pulses are observed. Thus it is always necessary to determine the frequency of each pulse. This is easily done by examining the base voltage of the Channel 3 signal, which is very close to $0.0 \mathrm{~V}$ for on-line pulses and about $1.2 \mathrm{~V}$ for off-line pulses.

The Channel 3 base voltage is measured in the Return Region near the center of the pulse. Once this value is determined, the remainder of the Channel 3 Return Region is redundant and need not be saved to a file. Also, one might opt to save only Good pulses, as described above. Thus, the use of some rudimentary DAP SW during data acquisition can decrease the data transfer and storage overhead. 
FFT1

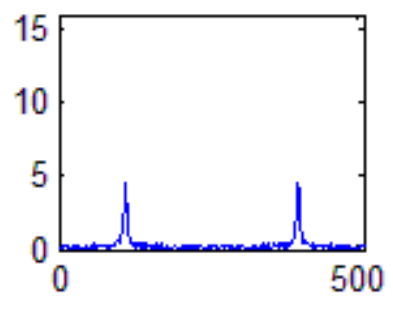

FFT2

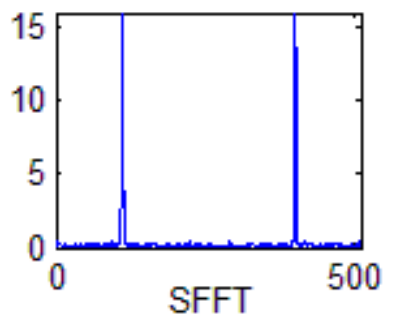

FFT3

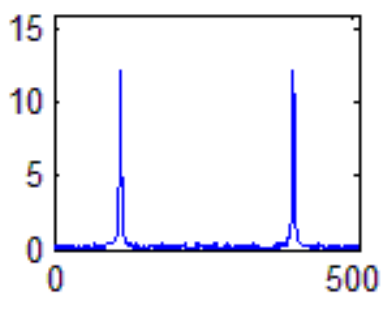

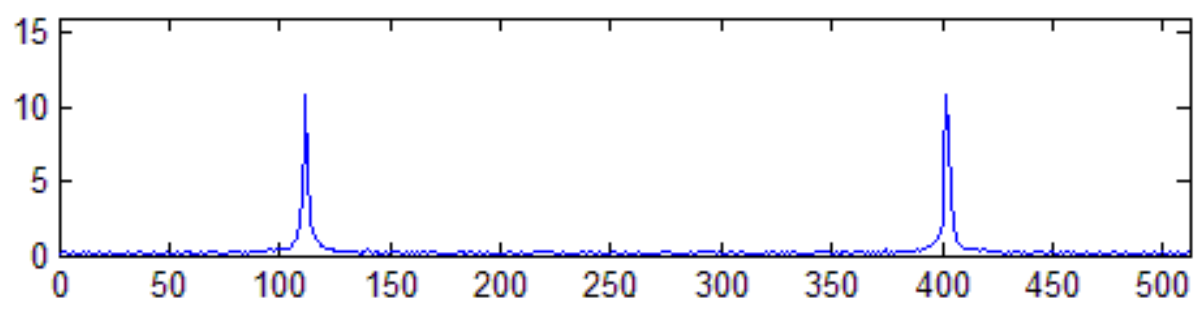

Fig. 4. Magnitudes of 50\% overlapping, 512-element FFTs (top row) of the Monitor Region of Shot \#1, Channel 1 . These are combined to get an average (bottom row) FFT. The peak frequency (at index 112 here, corresponding to about 109 $\mathrm{MHz}$ ) is used to determine if the return data is to be considered Good and further processed, or Bad and discarded.

\section{CONCLUSIONS}

DAQ SW was rapidly prototyped to demonstrate the ability to make very high frequency atmospheric $\mathrm{CO}_{2}$ measurements using a coherent DIAL lidar instrument previously used for coherent Doppler wind measurements. This CO2 Measurement Demonstration showed that the HW and SW could satisfy the challenging requirements related to sampling frequency and data storage for this application. There are additional techniques and digitizer configurations that could accommodate future, more taxing requirements.

It may be beneficial to develop DAQ SW for such dual-use lidars, especially if there is an anticipated need to support future space-borne versions of these instrument. While the DAP SW may be separately developed for post-processing, some DAP functionality is required during acquisition to provide quick-look display information, and to determine the quality of each return pulse. By filtering the data based on quality and retaining only what is acceptable, the challenging requirements for data storage and transfer posed by these instruments can be mitigated. 


\section{ACKNOWLEDGMENT}

This work was supported by the NASA Instrument Incubator Program, and the NASA Laser Risk Reduction Program.

\section{REFERENCES}

[1] Yu, J., Singh, U. N., Barnes, N. P., and Petros, M., 1998: “125-mJ diode-pumped injection-seeded Ho:Tm:YLF laser," Optics Letters 23, 780-782.

[2] Beyon, J. Y. and Koch, G. J.,“Novel Nonlinear Adaptive Doppler Shift Estimation Technique (NADSET) for the Coherent Doppler Lidar System VALIDAR,” in Proc. of the Defense and Security Symposium 2006 (62361), Orlando, FL, April, 2006.

[3] Beyon, J. Y. and Koch, G. J.,"Resolution Study of Wind Parameter Estimates by a Coherent Doppler Lidar System,” in Proc. of the Defense and Security Symposium 2006 (6214-3), Orlando, FL, April, 2006.

[4] Beyon, J. Y. and Koch, G. J.,"Wind Profiling by a Coherent Doppler Lidar System VALIDAR with a Subspace Decomposition Approach,” in Proc. of the Defense and Security Symposium 2006 (6236-5), Orlando, FL, April, 2006.

[5] Beyon, J. Y., Koch, G. J., and Li, Z., "Noise Normalization and Windowing Functions for VALIDAR in Wind Parameter Estimation,” in Proc. of the Defense and Security Symposium 2006 (6214-4), Orlando, FL, April, 2006.

[6] Beyon, J. Y. and Koch, G. J., “Novel nonlinear adaptive Doppler-shift estimation technique for the coherent Doppler validation lidar,” Optical Engineering, Vol. 46, No. 1, pp. 016002-1 - 016002-10, January 2007.

[7] Beyon, J. Y., Koch, G. J., and Ismail, S. "Signal processing techniques for heterodyne differential absorption lidar,” in Proc. of the Defense and Security Symposium 2007 (6567-53), Orlando, FL, April, 2007.

[8] Koch, G. J., Beyon, J. Y., Barnes, B. W., Petros, M., Yu, J., Amzajerdian, F., Kavaya, M. J., and Singh, U. N., "High-Energy 2- $\mu m$ Doppler Lidar for Wind Measurements," Optical Engineering, Vol. 46(11), pp. 116201-1 116201-14, November, 2007.

[9] Koch, G. J., Beyon, J. Y., F. Gibert, Barnes, B. W., Ismail, S., Petros, M., P.J. Petzar, Yu, J., Modlin, E. A., Davis, K. J., and Singh, U. N., 2008: "Side-line tunable laser transmitter for Differential Absorption Lidar measurement of $\mathrm{CO}_{2}$ : Design and application to atmospheric measurement," Appl. Opt. 47, 944-956.

[10] Beyon, J. Y., Koch, G. J., Kavaya, M. J., and Sahota, M., "Comparison of theoretical and empirical statistics of wind measurements with validation lidar (VALIDAR)," in Proc. of the Defense and Security Symposium 2008(6968-59), Orlando, FL, March, 2008.

[11] Demoz, B., "The Howard University Beltsville Research Campus: Highlights from the Recent Wind Lidar and Water Experiments,” Wind Lidar Working Group Meeting, June, 2009.

[12] Koch, G. J., "Ground-Based Testing of the DAWN 2-Micron Doppler Lidar and Comparison with Other Sensors," Wind Lidar Working Group Meeting, June, 2009.

[13] Vermeesch, K., Koch, G., Gentry, B., Bacha, T., Chen, H., and Demoz, B., "Comparisons of Ground-Based, Radiosonde, and Aircraft Wind Measurements at the Howard University Beltsville Research Site,” Wind Lidar Working Group Meeting, June, 2009.

[14] Agilent Technologies, Programmer's Reference Manual Agilent Acqiris Instruments, Edition I-RevA, February 2009.

[15] Agilent Technologies, User Manual Agilient Acqiris 10-bit Digitizers, Edition B-RevH, February 2009. 